\title{
ESTIMATIVA DO COMPORTAMENTO DO FOGO EM QUEIMAS EXPERIMENTAIS SOB POVOAMENTOS DE Pinus elliottii ${ }^{1}$
}

\author{
Antonio Carlos Batista ${ }^{2}$, Alexandre Beutling ${ }^{3}$ e José Fernando Pereira ${ }^{4}$
}

\begin{abstract}
RESUMO - Foram realizadas 23 queimas experimentais em um talhão de Pinus elliottii da Estação Experimental de Rio Negro-PR, município de Rio Negro, pertencente à Universidade Federal do Paraná, durante o inverno de 2008. O objetivo desta pesquisa foi desenvolver modelos de previsão do comportamento do fogo para o planejamento de prevenção e combate aos incêndios florestais. Nos locais preparados paras as queimas foram efetuadas observações e medições sobre o material combustível e variáveis meteorológicas e, durante as queimas, sobre o comportamento do fogo: velocidade de propagação, intensidade do fogo e dimensões da chama. Correlacionando-se os dois grupos de variáveis (do ambiente versus variáveis do comportamento do fogo), obtiveram-se associações significativas entre estas variáveis. Os modelos de regressão desenvolvidos através dos dados coletados produziram estimativas estatisticamente confiáveis do comportamento do fogo em termos de consumo de material combustível, temperatura do ar, umidade relativa e velocidade do vento. Também se verificou que a altura da chama é uma variável fortemente associada à intensidade do fogo, podendo ser empregada para estimativa desta nas condições em que o experimento foi realizado.
\end{abstract}

Palavras-chave: Comportamento do fogo; Queimas controladas; Incêndios florestais; Combustíveis florestais.

\section{FIRE BEHAVIOR EVALUATION IN EXPERIMENTAL FIRES ON FOREST FUELS OF Pinus elliottii PLANTATIONS}

\begin{abstract}
A series of 23 experimental fires was carried out in a Pinus elliottii forest fuels plantation of the Experimental Station of Rio Negro-PR, Rio Negro, Universidade Federal do Paraná, in July 2008. The objective of this research was to develop fire behavior prediction models for fire management purposes. Observations and measurements of the forest fuels and meteorological conditions were carried out in the local prepared for the fires and fire behavior characteristics (fire spread, fire intensity and flame height)were measured during the fires. By correlating both groups of variables (environmental versus fire behavior) it was obtained significant association between these variables. Regression models developed using the collected data are able to predict fire behavior in terms of forest fuel consumption, air temperature, relative humidity and wind speed. It was also observed that the flame height is strongly associated with fire intensity and it can be used to estimate this intensity in the conditions which the data were collected.
\end{abstract}

Keywords: Controlled burning; Fire behavior; Forest fires; Forest fuels.

\footnotetext{
${ }^{1}$ Recebido em 20.03.2009 aceito para publicação em 13.08.2013.

${ }^{2}$ Universidade Federal do Paraná, Brasil. E-mail: <batistaufpr@ufpr.br>.

${ }^{3}$ Universidade Federal de Mato Grosso do Sul, Brasil. E-mail: <a.beutling@ufms>.

${ }^{4}$ Programa de Pós-graduação em Engenharia Florestal na Universidade Federal do Paraná, Brasil. E-mail:

<ultimo.jose@yahoo.com.br>.
} 


\section{1- INTRODUÇÃO}

Os incêndios florestais são importantes causadores de danos às florestas, especialmente os plantios florestais.

Os efeitos produzidos pelo fogo em determinado lugar dependem do seu comportamento, que é função das características da floresta e dos fatores ambientais. Comportamento do fogo é um termo genérico utilizado para descrever as principais características da combustão em um incêndio florestal ou uma queima, e refere-se à maneira como o material entra em combustão, como se desenvolvem as chamas e como o fogo se propaga e apresenta outros fenômenos.

O estudo do comportamento do fogo tem possibilitado entender os fatores que têm um papel importante no início, na propagação e na dificuldade de se extinguir os incêndios. Basicamente, as variáveis que descrevem o comportamento do fogo são: velocidade de propagação, intensidade da linha de fogo, taxa de energia liberada e tempo de residência. Essas variáveis quantificam e caracterizam o comportamento do fogo, bem como controlam a dificuldade de extinção de qualquer incêndio (LENTILE, 2006). Outras variáveis como temperaturas máximas alcançadas nas zonas de combustão e altura de crestamento letal, além de descrever aspectos do comportamento do fogo, possibilitam estabelecer associações com o efeito produzido nos diversos elementos do ecossistema florestal (SOARES; BATISTA, 2007).

Diversas pesquisas têm sido realizadas em muitos países desde meados do século passado, relacionando as características dos combustíveis e as condições meteorológicas com as variáveis do comportamento do fogo, com o objetivo de estabelecer modelos de previsão das características dos incêndios e queimadas e os seus efeitos potenciais sobre o ambiente florestal (ROTHERMEL, 1972; BURGAN; ROTHERMEL, 1984; ANDREWS, 1986; CATCHPOLE et al., 1998; VEGA et al., 1998; FERNANDES, 2001). Muitos destes experimentos serviram de base para a elaboração de simuladores do comportamento do fogo que são utilizados largamente no manejo do fogo em florestas e plantios florestais, como por exemplo, os softwares Behaveplus (2008) nos Estados Unidos da América, o CARDIN (VELÈZ, 2000) na Espanha e o KITRAL (JULIO, 1996) no Chile. O uso destes softwares, no entanto, deve se restringir aos ambientes similares às regiões onde os experimentos foram desenvolvidos, devido a grande diversidade dos ambientes e a consequente variabilidade do comportamento do fogo.

O desenvolvimento de modelos empíricos de comportamento do fogo continua sendo fundamental para compreender a relação entre o fogo e o ambiente em diversos ecossistemas florestais e servem como instrumentos fundamentais no manejo do fogo. Em muitos países, os modelos de estimativa do comportamento do fogo continuam sendo desenvolvidos empiricamente a partir de experimentos de queima (BILGILI; SAGLAM, 2003; KÜÇÜK et al; 2008; MENDES-LOPES et al., 2003; FERNANDES; 2004).

No Brasil, há mais de 20 anos se desenvolvem pesquisas sobre comportamento do fogo em reflorestamentos (SOARES et al., 2009, BATISTA e NUNES, 2009). Nestas pesquisas, o comportamento do fogo é avaliado mediante os parâmetros velocidade de propagação e intensidade do fogo.

O objetivo desta pesquisa foi medir os parâmetros do comportamento do fogo em queimas experimentais em condições de campo, comparando-os com as características ambientais de um reflorestamento de Pinus elliottii durante as queimas, estabelecer correlações entre as variáveis do fogo e os fatores do ambiente e elaborar modelos para estimativa do comportamento do fogo.

\section{MATERIAL E MÉTODOS}

Para a realização desta pesquisa foi instalado um experimento de queima controlada em um povoamento de Pinus elliottii, com 12 anos de idade, da Estação Experimental de Rio Negro, pertencente à Universidade Federal do Paraná, localizada no município de Rio NegroPR. Durante o mês julho de 2008 foram monitoradas as condições meteorológicas (temperatura, umidade relativa e número de dias sem chuva) para definição dos dias adequados para realização das queimas.

Em cada uma das datas escolhidas realizaram-se queimas de 23 parcelas previamente estabelecidas e preparadas.

Em cada parcela, com dimensão de 60 m² (3 m x 20 m), foram efetuadas observações e medições sobre o material combustível, declividade do terreno, variáveis meteorológicas e, durante as queimas, sobre o comportamento do fogo. 
Para a caracterização do material combustível foram obtidas informações sobre o tipo, a quantidade, o conteúdo de umidade e a espessura formada pela deposição do material sobre o solo. Esses dados foram obtidos através do inventário do material combustível depositado no piso da floresta. Para isso, foram alocadas aleatoriamente em cada parcela, 3 amostras de $400 \mathrm{~cm}^{2}(20 \mathrm{~cm}$ x $20 \mathrm{~cm})$ cada uma, de onde o material foi coletado separadamente em 4 categorias: acículas, material com diâmetro entre 0 e 0,7 cm, material com diâmetro entre 0,71 e 2,5 cm e material com diâmetro entre 2,51 e 7,6 cm. Esse material foi colocado em estufa a $75^{\circ} \mathrm{C}$ até peso constante para determinação do peso de matéria seca. O inventário do material combustível foi feito antes e depois de cada queima. A diferença observada entre a quantidade antes (combustível total) e depois da queima (combustível residual) forneceu a quantidade de material consumida pela queima (combustível disponível). A espessura da camada formada pelo material depositado no piso florestal foi feita nas amostras do inventário do material combustível e após as queimas, mediu-se novamente a espessura do material acamado restante (combustível residual). A diferença entre a espessura da manta antes e depois da queima possibilitou estimar a variável "profundidade da queima” (PQ). Para determinação do conteúdo de umidade do material combustível foram coletadas cinco amostras representativas de cada classe de material combustível antes do início de cada queima. Essas amostras foram pesadas e colocadas em estufas a $75^{\circ} \mathrm{C}$ até peso constante para determinação de matéria seca. Pela diferença entre o peso inicial e o de matéria seca das amostras foi estimado o conteúdo de umidade do combustível.

Durante o período do experimento, as condições meteorológicas foram monitoradas continuamente através de um termohigrógrafo e de um pluviógrafo instalados próximos (cerca de 100 metros) do local das queimas. Durante as queimas também foram feitas observações meteorológicas no interior do povoamento, junto às parcelas que foram queimadas. Foram observadas a temperatura, a umidade relativa do ar e a direção e velocidade do vento. As medições foram feitas continuamente por uma estação meteorológica portátil marca Watchdog 2000 series, com datalogger para armazenamento dos dados, e ajustada para leitura das variáveis temperatura, umidade relativa, velocidade e direção do vento, de minuto em minuto, e também com instrumento de leitura direta (Kestrel 3000 Pocket Weather Station).
No momento em que estava ocorrendo a queima de cada parcela, foram feitas observações sobre as seguintes variáveis do comportamento do fogo, conforme procedimentos padrão adotados internacionalmente e recomendados por diversos autores (KUÇUK et al., 2008; RIBEIRO et al., 2006; GARNICA et al., 2006):

- Velocidade de propagação - obtida visualmente, determinando-se o tempo necessário para a linha de fogo percorrer distâncias de 2 metros, previamente demarcadas em cada parcela, no sentido do seu comprimento (20 m);

- Altura das chamas - estimativa visual da altura média alcançada pelas chamas a cada metro de avanço da linha de fogo, com o auxílio de comparadores (escalas) de dimensões conhecidas;

- Intensidade do fogo - estimada pela equação proposta por Byram (1959):

$$
I=H \cdot w \cdot r
$$

sendo:

$\mathrm{I}=$ intensidade do fogo em $\mathrm{kw} \cdot \mathrm{m}^{-1}$

$\mathrm{H}=$ poder calorífico em $\mathrm{kj} \cdot \mathrm{kg}^{-1}$

$\mathrm{w}=$ peso do combustível disponível em kg.m-2

$\mathrm{r}=$ velocidade de propagação do fogo em m. $\mathrm{s}^{-1}$

Utilizou-se $\mathrm{H}=15490$ kj.kg-1 (poder calorífico) como valor médio para o material combustível de Pinus elliotii, conforme determinações feitas por Soares e Hakkila(1987).

A partir da coleta e processamento dos dados foi possível construir uma matriz composta de variáveis ambientais (combustível e condições meteorológicas) e variáveis do comportamento do fogo. As variáveis utilizadas no presente estudo estão apresentadas e descritas na tabela 1 .

Os dados obtidos, referentes ao ambiente (material combustível e condições meteorológicas) e ao comportamento do fogo, foram processados em planilhas (Microsoft Office Excel 2003) e analisados através do software STATGRAPHICS Plus for Windows Versão 4.1. Os testes estatísticos utilizados para análise e comparação dos dados foram: análise de correlação, análise de regressão múltipla e análise de variância.

Revista Árvore, Viçosa-MG, v.37, n.5, p.779-787, 2013 
Tabela 1 - Variáveis ambientais e do comportamento do fogo, obtidas durante a realização das queimas e utilizadas para compor a matriz de dados do experimento.

Table 1 - Environmental and fire behavior variables, obtained during experimental fires and used for composing data matrix of the experiment.

\begin{tabular}{|c|c|c|}
\hline Variável & Descrição & Unidade \\
\hline ESPA & Espessura da manta de combustível antes da queima da parcela (média de 3 parcelas) & $\mathrm{cm}$ \\
\hline ESPD & Espessura da manta de combustível depois da queima da parcela (média de 3 parcelas) & $\mathrm{cm}$ \\
\hline Hch & Altura das chamas durante a queima (média de 10 observações por parcela) & $\mathrm{cm}$ \\
\hline Hora & Hora de início da queima & $\mathrm{H}$ \\
\hline MCA & Peso do material combustível antes da queima (média de 3 parcelas) & kg. $\mathrm{m}^{-2}$ \\
\hline MCD & Peso do material combustível depois da queima (média de 3 parcelas) & kg. $\mathrm{m}^{-2}$ \\
\hline Mccons & Material combustível consumido pelo fogo & kg. $\mathrm{m}^{-2}$ \\
\hline Período & Período do dia em que foi realizada a queima & 1 a $4^{1}$ \\
\hline PQ & Profundidade de queima & $\mathrm{cm}$ \\
\hline $\mathrm{T}$ & Temperatura do ar no início da queima da parcela & ${ }^{\circ} \mathrm{C}$ \\
\hline UR & Umidade relativa do ar no início da queima da parcela & $\%$ \\
\hline Velprop & Velocidade de propagação do fogo (média de 10 observações por parcela) & m. $\min ^{-1}$ \\
\hline Vv & Velocidade do vento no início da queima da parcela & $\mathrm{m} \cdot \mathrm{s}^{-1}$ \\
\hline Dv & Direção do vento no início da queima da parcela & Graus \\
\hline Int & Intensidade do fogo (média de 10 observações por parcela) & kw.m ${ }^{-1}$ \\
\hline
\end{tabular}

1 - Dividiu-se os dias das queimas em 4 períodos: 1 (antes das 10 horas da manhã), 2 (10 às 12 horas), 3 (12 às 14 horas) e 4 (14 às 16 horas).

Os modelos de regressão foram ajustados no software STATGRAPHICS Plus for Windows Versão 4.1, através da função "Regression Model Selection" e seleção dos melhores modelos foi feita pelo maior valor do coeficiente de determinação $\left(\mathrm{R}^{2}\right)$ e pelo coeficiente estatístico Mallows Cp. A estatística Cp de Mallows é delineada contra o número de parâmetros (p); quanto mais próximo for Cp de $\mathrm{p}$, menos tendenciosas são as estimativas dos parâmetros e melhor é o modelo (FLORIANO et al., 2006).

\section{RESULTADOS}

Na tabela 2 encontra-se o sumário das observações feitas durante a realização das queimas. Das 23 queimas realizadas, foram utilizados dados de apenas 13 , descartando-se os demais devido à inconsistência de algumas observações, problemas técnicos durante a realização das queimas (mudança na direção do vento, por exemplo) e descontinuidade da linha de fogo.

Pode-se verificar que as condições meteorológicas se mantiveram relativamente homogêneas durante os dias de queima, com temperaturas médias variando entre 20 e $23^{\circ} \mathrm{C}$, umidade relativa entre 50 e $70 \%$ e vento calmo (até $1,1 \mathrm{~m} \cdot \mathrm{s}^{-1}$ ). Embora tenha sido selecionada uma área com características ambientais aparentemente homogêneas, pode-se observar que houve variação nas características do material combustível (peso e espessura da manta) entre as parcelas. Os valores médios encontrados, no entanto, são similares aos encontrados em outras pesquisas dessa natureza no sul do Brasil (BATISTA, 1995; KOEHLER, 1989).

As variáveis do comportamento do fogo também apresentaram variação expressiva, característica da combustão em ambiente florestal, como se pode verificar em diversos experimentos de queima em que se fizeram medições dos parâmetros do fogo (RIBEIRO; SOARES, 1999; SANTONI et al., 2006; VEGA et al., 2006).

Na tabela 3 estão apresentadas as correlações obtidas entre todas as variáveis estudadas.

Dos resultados da Tabela 3, é importante destacar as correlações da variável meteorológica "UR" com as demais variáveis. Observa-se que essa variável apresentou correlações significativas com a maioria das variáveis analisadas.

Dentre as variáveis que descrevem o material combustível destacam-se as variáveis "ESPA" e "ESPD” que apresentaram correlações significativas com as demais variáveis ambientais e do comportamento do fogo.

As maiores correlações entre as variáveis do comportamento do fogo ocorreram para "Hch” X "Velprop” ( $r=0,862)$, seguida da correlação entre Int X Hch $(r=0,7774)$. A variável "Int” também apresentou 
Tabela 2 - Sumário estatístico das variáveis ambientais e do comportamento do fogo durante as queimas experimentais realizadas em um plantio de Pinus elliottii na Estação Experimental de Rio Negro, PR.

Table 2 - Statistic summary of environmental and fire behavior variables during the experimental fires on Pinus elliotii plantation at the Experimental Station of Rio Negro, PR.

\begin{tabular}{llcccc}
\hline Variável & $\mathrm{N}$ & Média & Desvio Padrão & Valor mínimo & Valor máximo \\
\hline ESPA & 13 & 11,01 & 1,7011 & 8,02 & 13,0 \\
ESPD & 13 & 8,00 & 2,0521 & 5,33 & 9,67 \\
Hch & 13 & 0,196 & 0,0611 & 0,1 & 0,33 \\
Hora & 13 & 13,3 & 1,5221 & 11,5 & 6,83 \\
MCA & 13 & 5,282 & 0,9061 & 3,14 & 6,22 \\
MCD & 13 & 4,401 & 0,9717 & 3,21 & 5,08 \\
Mccons & 13 & 1,641 & 1,328 & 0,081 & 4,17 \\
Período & 13 & 2,77 & 1,423 & 1,0 & 23,6 \\
PQ & 13 & 3,0 & 0,8634 & 20,0 & 70,0 \\
T & 13 & 21,82 & 1,364 & 54,4 & 0,180 \\
UR & 13 & 63,75 & 5,2157 & 0,086 & 1,1 \\
Velprop & 13 & 0,139 & 0,0276 & 0,0 & 2,0 \\
Vv & 13 & 0,25 & 0,31 & 2,5324 & 2,321 \\
Int & 13 & 63,791 & 63,7508 & & \\
\hline
\end{tabular}

Tabela 3 - Correlações entre as variáveis ambientais e as variáveis do comportamento do fogo durante a realização das queimas sob os plantios de Pinus elliotii na Estação Experimental de Rio Negro, PR.

Table 3 - Correlations between environmental and fire behavior variables during the experimental fires on Pinus elliotii plantation at the Experimental Station of Rio Negro, $P R$.

\begin{tabular}{|c|c|c|c|c|c|c|c|c|c|c|c|c|c|}
\hline & ESPA & ESPD & Hch & Hora & MCA & Mccons & MCD & Período & PQ & $\mathrm{T}$ & UR & Velprop & $\mathrm{Vv}$ \\
\hline ESPD & $0,8618 * *$ & & & & & & & & & & & & \\
\hline Hch & 0,4252 & $0,6277^{*}$ & & & & & & & & & & & \\
\hline Hora & 0,5599* & 0,4996 & $-0,0494$ & & & & & & & & & & \\
\hline MCA & 0,2873 & 0,1068 & $-0,2555$ & 0,3463 & & & & & & & & & \\
\hline Mccons & $-0,1146$ & $-0,0703$ & 0,6738* & $-0,5507 *$ & $-0,3628$ & & & & & & & & \\
\hline MCD & 0,6298* & 0,5916* & 0,3192 & 0,4485 & 0,4237 & $-0,0684$ & & & & & & & \\
\hline Período & 0,5869* & 0,5197 & $-0,0110$ & 0,9918** & 0,2882 & $-0,4989$ & 0,4935 & & & & & & \\
\hline PQ & 0,5404 & 0,0389 & $-0,2035$ & 0,2742 & 0,3950 & $-0,1092$ & 0,2593 & 0,2956 & & & & & \\
\hline $\mathrm{T}$ & 0,1076 & 0,1917 & 0,4444 & $-0,0596$ & $-0,3474$ & 0,4215 & 0,1414 & 0,0201 & $-0,1061$ & & & & \\
\hline UR & $-0,5795 *$ & $-0,7845^{* *}$ & $-0,6873^{*}$ & $-0,5428$ & $-0,1083$ & $-0,0427$ & $-0,5144$ & $-0,5315$ & 0,1596 & 0,0505 & & & \\
\hline Velprop & 0,4156 & $0,6471^{*}$ & $0,8620 * *$ & $-0,1623$ & $-0,4499$ & 0,5258 & 0,0976 & $-0,1174$ & $-0,2548$ & 0,4712 & $-0,4574$ & & \\
\hline $\mathrm{Vv}$ & $-0,0905$ & $-0,0678$ & 0,0824 & $-0,6287^{*}$ & $-0,4039$ & 0,2085 & $-0,2669$ & $-0,6065 *$ & $-0,0659$ & $-0,1497$ & 0,3583 & 0,3455 & \\
\hline Int & 0,0447 & 0,1068 & $0,7774 * *$ & $-0,4756$ & $-0,3628$ & $0,9764 * *$ & 0,0264 & $-0,4125$ & $-0,0892$ & 0,4717 & $-0,1487$ & $0,6735^{*}$ & 0,2698 \\
\hline
\end{tabular}
correlation at $99 \%$ probability level; * Significant correlation at $95 \%$ probability level.

alta correlação com as variáveis “Mccons” e "Velprop”. No entanto, isto foi devido a variável "Int” ter sido calculada a partir destas duas variáveis pela fórmula de Byram ( Int = H x Velprop x Mccons).

Na tabela 4 estão apresentados os modelos de regressão para estimativa das variáveis do comportamento do fogo em função das variáveis ambientais e características dos combustíveis. Foram ajustados 1024 modelos pelas diferentes combinações das variáveis ambientais e dos combustíveis para estimativa da altura das chamas (Hch). As variáveis mais importantes para estimativa da altura das chamas foram: o consumo do material combustível (Mccons), a temperatura e umidade relativa do ar e a velocidade do vento. Para a estimativa da velocidade de propagação do fogo também foram ajustados 1024 modelos usando-se os mesmos procedimentos anteriores. Nesse caso também as variáveis mais importantes para estimativa da velocidade de propagação do fogo foram: umidade relativa, temperatura do ar e velocidade do vento. Para estimativa da 
intensidade do fogo ajustou-se 512 modelos. As variáveis independentes mais importantes para estimativa da intensidade do fogo foram: a altura das chamas (Hch) e a espessura da manta após a queima (ESPA).

\section{DISCUSSÃO}

Devido a grande variabilidade do comportamento do fogo em função da direção do vento, neste estudo só foram utilizados dados das parcelas em que a linha de fogo avançava na direção do vento. Tecnicamente, o estudo restringiu-se ao comportamento do fogo em queimas a favor do vento.

Os experimentos sobre o comportamento do fogo em ambiente natural aberto são difíceis de serem realizados devido à dificuldade em controlar e/ou monitorar o grande número de variáveis envolvido no processo de combustão (Figura 1). No entanto, esse é um dos meios indispensáveis para o entendimento das relações entre o ambiente florestal e a combustão, possibilitando o estabelecimento das bases para a previsão do comportamento do fogo nas áreas florestais do Brasil.

Uma das variáveis ambientais de grande importância na avaliação do comportamento do fogo é a topografia do terreno, particularmente a inclinação, que não foi avaliada neste trabalho, devido o local onde se realizou as queimas ser plano. No entanto, a contribuição desta variável é fundamental na elaboração de modelos de previsão do comportamento do fogo e deve ser computada sempre que for possível.

Confrontando os resultados de comportamento do fogo obtidos neste estudo com pesquisas similares realizadas em outras regiões, pode-se constatar que há concordância em diversos aspectos, embora os ambientes e o tipo de vegetação sejam diferentes.

Um dos resultados mais relevantes dessa pesquisa, para a estimativa do comportamento do fogo, foi a alta correlação obtida entre a altura das chamas e as variáveis ambientais e as características dos combustíveis. De acordo com Byram (1959), a intensidade do fogo pode ser estimada através do comprimento das chamas. Nesse experimento optou-se por medir a altura ao invés do comprimento das chamas, devido a maior facilidade de obtenção da altura das chamas em ambiente aberto. Diversas pesquisas têm evidenciado que a dimensão, da chama que apresenta melhor associação com as demais variáveis do comportamento do fogo é o comprimento das chamas (MENDES-LOPES et al., 2003; BATISTA et al., 1993) . No entanto, a altura da chama ("Hch”) refletiu bem a variação do comportamento do fogo em função das condições ambientais e dos combustíveis florestais neste experimento, como se pode constatar pelos modelos apresentados na Tabela 4.

Tabela 4 - Modelos de regressão para estimativa do comportamento do fogo em queimas controladas sob plantios de Pinus elliottii, na Estação Experimental de Rio Negro, PR.

Table 4 - Regression models for estimating fire behavior in experimental fires on Pinus elliotii plantation at the Experimental Station of Rio Negro, PR.

\begin{tabular}{|c|c|c|c|}
\hline Altura das chamas & $\mathrm{R}^{2}$ ajustado & $\mathrm{S}_{\mathrm{yx}}$ & $\mathrm{F}$ \\
\hline \multicolumn{3}{|l|}{ Hch $=0,338628+0,00570537 *$ MCA $+0,0205005 *$ Mccons $+0,0167875 * \mathrm{~T}-$} & $69,89 * *$ \\
\hline Hch $=0,398185+0,0200958 *$ Mccons $+0,0153596 * T-0,00920763 * U R+$ & & & \\
\hline $0,0434501 * \mathrm{Vv}$ & 96,3562 & 0,0116773 & $80,33 * *$ \\
\hline Hch $=0,41209+0,02489 *$ Mccons $+0,0112004 * \mathrm{~T}-0,00786175 * \mathrm{UR}$ & 88,5749 & 0,0206776 & $32,01 * *$ \\
\hline Hch $=0,635419+0,0297738 *$ Mccons $-0,00765657 *$ UR & 83,5936 & 0,0247786 & $31,57 * *$ \\
\hline Velocidade de propagação do fogo & $\mathrm{R}^{2}$ ajustado & $\mathrm{S}_{\mathrm{yx}}$ & $\mathrm{F}$ \\
\hline \multicolumn{4}{|l|}{ Velprop $=0,164336-0,00739535 * \mathrm{MCD}+0,0132841 * \mathrm{~T}-0,00470706 * \mathrm{UR}+$} \\
\hline $0,0467416 * \mathrm{Vv}$ & 87,6613 & 0,00972863 & $22,31 * *$ \\
\hline Velprop $=0,104716+0,0124587 * \mathrm{~T}-0,00400681 * \mathrm{UR}+0,0478819 * \mathrm{Vv}$ & 82,7309 & 0,0115094 & $20,16 * *$ \\
\hline Intensidade do fogo & $\mathrm{R}^{2}$ ajustado & $\mathrm{S}_{\mathrm{yx}}$ & $\mathrm{F}$ \\
\hline \multicolumn{4}{|l|}{ Int $=822,92-20,6402 *$ ESPA $+1070,72 *$ Hch $-78,5972 *$ Hora +} \\
\hline $74,0037 *$ Periodo $+32,7532 * P Q$ & 90,697 & 19,4445 & $24,40 * *$ \\
\hline Int $=-375,134-19,7783 * E S P A+1388,14 * H c h+30,1247 * P Q+4,61088 * U R$ & 88,0881 & 22,0027 & $23,18 * *$ \\
\hline Int $=10,1062-30,3176 * \mathrm{ESPA}+1296,18 * \mathrm{Hch}+44,3797 * \mathrm{PQ}$ & 87,7203 & 25,7958 & $21,43 * *$ \\
\hline Int $=-718,028+1275,87 *$ Hch $+8,33757 * \mathrm{UR}$ & 85,2354 & 26,8341 & $28,86 * *$ \\
\hline
\end{tabular}

Revista Árvore, Viçosa-MG, v.37, n.5, p.779-787, 2013 

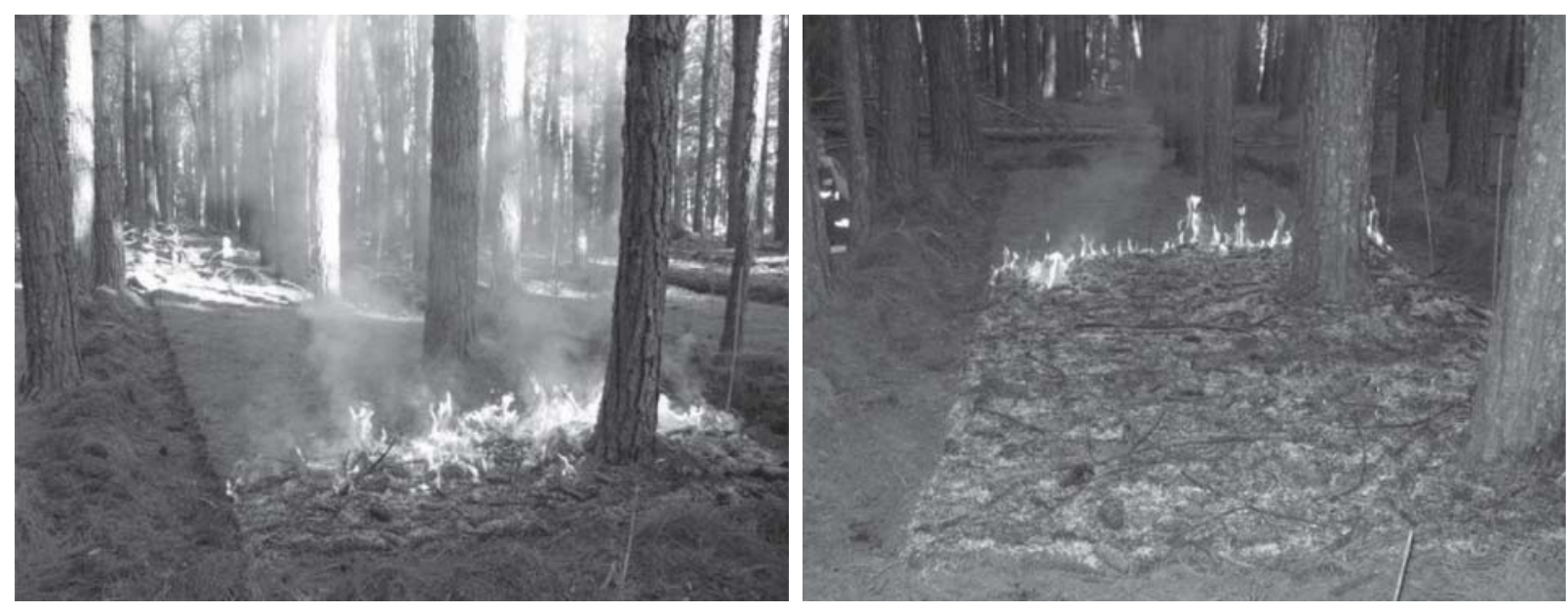

Figura 1 - Aspectos do comportamento do fogo em queimas experimentais sob povoamentos de Pinus elliottii na Estação Experimental de Rio Negro, PR.

Figure 1 - Fire behavior aspects in experimental fires on Pinus elliotii plantations, Experimental Station of Rio Negro, PR.

A velocidade de propagação do fogo é um dos parâmetros mais difíceis de ser estimado, devido a diversidade de fatores ambientais associados à propagação do fogo. Diversas pesquisas têm sido realizadas visando encontrar um modelo ideal para descrever a taxa de propagação do fogo em combustíveis florestais. Até este momento, o modelo genérico mais aceito é o proposto por Rothermel (1972). No entanto, esse modelo é difícil de ser empregado devido ao grande número de variáveis utilizadas e à dificuldade de obtenção destas em incêndios e queimas experimentais. Por isso, modelos empíricos de estimativa da velocidade de propagação do fogo têm sido desenvolvidos em várias regiões do mundo, embora sejam de uso limitado a condições específicas, tanto de ambiente como de características do fogo. A estimativa da velocidade de propagação do fogo ("Velprop”), a partir das variáveis meteorológicas T, UR e Vv, apresentou um desempenho semelhante ao observado por Bilgili e Salam (2003), Vega et al. (2006) e Davies et al. (2006) em experimentos de queima sob povoamentos florestais. É importante ressaltar que a velocidade do vento é a variável obrigatoriamente incluída na maioria dos modelos empíricos de estimativa de velocidade de propagação do fogo.

A intensidade do fogo é a variável mais importante do comportamento do fogo. É a variável que descreve a magnitude da combustão em termos de energia libertada. Por isso também é o parâmetro mais empregado para comparar queimas e incêndios florestais, bem como é usada como parâmetro para avaliar os efeitos do fogo no ambiente. Os modelos para estimativa da intensidade do fogo apresentados na Tabela 2 mostram a forte associação entre a intensidade e altura da chamas (Hch), comprovando o fato observado por vários autores (DAVIS, 1959; BATISTA et al., 1993; MENDES-LOPES et al., 2003) de que é possível obter uma boa estimativa da intensidade do fogo através das dimensões das chamas.

\section{CONCLUSÕES}

As observações sobre o comportamento do fogo, sobre as condições meteorológicas e sobre as características dos combustíveis, durante as queimas controladas realizadas sob um povoamento de Pinus elliottii na Estação de Experimental de Rio Negro permitem concluir que:

- A avaliação do comportamento do fogo em ambiente natural aberto é difícil de ser realizada devido a influência da interação e diversidade dos fatores associados à ignição e propagação do fogo;

-as variáveis do comportamento do fogo apresentaram correlações significativas com as variáveis que descrevem o combustível e as condições meteorológicas, viabilizando o desenvolvimento de modelos para estimativa do comportamento do fogo;

Revista Árvore, Viçosa-MG, v.37, n.5, p.779-787, 2013 
- as variáveis “Mccons” e "ESPA” que descrevem as características dos combustíveis, juntamente com as variáveis meteorológicas T, UR e Vv, foram as que melhor explicam a variação do comportamento do fogo;

- pelos resultados obtidos, pode-se afirmar que a variável “Hch” é um parâmetro confiável para estimativa da intensidade do fogo.

\section{REFERÊNCIAS}

ANDREWS, P. L. Behave: fire behavior prediction and fuel modeling system - BURN subsystem. Part 1. Ogden: USDA Forest Service, General Technical Report INT-194, 1986. 130p.

BAtistA, A. C. Avaliação da queima controlada em povoamentos de Pinus taeda L. no norte do Paraná. 1995. $188 \mathrm{f}$. Tese (Doutorado em Engenharia Florestal) Universidade Federal do Paraná, Curitiba, 1995.

BATISTA, A. C.; LIMA, G. S.; SOARES, R. V. Altura de carbonização da casca das árvores como estimador da intensidade do fogo em queima controlada. In: CONGRESSO FLORESTAL BRASILEIRO, 7., 1993, Curitiba; CONGRESSO FLORESTAL PANAMERICANO, 1., 1993, Curitiba. Anais... Curitiba: SBS-SBEF, 1993. p.169-171.

BEHAVEPLUS Fire Modeling System Version 4.0.0. Acesso em março de 2008. Disponível em: http:// www.firemodels.org/content/view/12/26/

BILGILI, E.; SAGLAM, B. Fire behavior in maquis fuels in Turkey. Forest Ecology and

Management, v.184, p.201-207, 2003.

BURGAN, R. E.; ROTHERMEL, R. C. Behave: fire behavior prediction and fuel modeling system - FUEL subsystem. Ogden: USDA Forest Service, General Technical Report INT-167, 1984. 126p.

BYRAM, G. M. Combustion of forest fuels. In: DAVIS, K. P. Forest fire: Control and use. New York: Mc Graw Hill, 1959. p.77-84.

CATCHPOLE, W. R. et al. Cooperative development of equations for heathland fire behaviour. In: VIEGAS, D. X., ed. In: INTERNATIONAL CONFERENCE ON FOREST FIRE RESEARCH, 3., Luso, 1998. Proceedings... Coimbra: University of Coimbra, 1998. p.631-45.
DAVIES G. M. et al. Developing shrub fire behaviour models in an oceanic climate: Burning in the British Uplands. In: VIEGAS, D. X., ed. In: INTERNATIONAL CONFERENCE ON FOREST FIRE RESEARCH, 5., Figueira da Foz, 2006. Proceedings... Portugal: University of Coimbra, 2006. p.S107.

DAVIS, K. P. Forest fire: Control and use. New York: McGraw Hill, 1959. p.77-84.

FERNANDES, P. A. M.; LOUREIRO, C. A.; BOTELHO, H. S. Fire behavior and severity in a maritime pine stand under differing fuel conditions. Annals of Forest Science., v.61, p.537-544, 2004.

FERNANDES, P. M. Fire spread prediction in shrub fuels in Portugal. Forest Ecology and Management, v.144, n.1, p.67-74, 2001.

FLORIANO, E. P. et al. Ajuste e seleção de modelos tradicionais para série temporal de dados de altura de árvores fitting and selecting traditional models for tree's height time series data. Ciência Florestal, v. 16, n. 2, p. 177 199, 2006.

GARNICA, J. G. F.; GONZALEZ, D. A. M.; SOLORIO, J. D. B. Forest fire behavior in prescribed burns under different environmental conditions in México. Forest Ecology and Management., v. 234, S1, p.131, 2006.

JULIO, G. Comportamiento del fuego: modelos de simulacion y su uso en actividades de combate. In: REUNIÃO TÉCNICA CONJUNTA FUPEF/SIF/ IPEF, 4., 1996, Curitiba; CURSO DE ATUALIZAÇÃO EM CONTROLE DE INCÊNDIOS FLORESTAIS, 2., 1996, Curitiba. Anais... Curitiba: FUPEF, 1996. p.1117-1129.

KOEHLER, C. W. Variação estacional da serapilheira e de nutrientes em povoamentos de Pinus taeda na região de Ponta Grossa-PR. 1989. 148f. Tese (Doutorado em Engenharia Florestal) - Universidade Federal do Paraná, Curitiba, 1989.

KÜÇÜK, O. et al. Some parameters affecting fire behavior in anatolian black pine slash Turkish Journal of Agriculture and Forestry, v.32, n.1, 121-129, 2008. 
LENTILE, L. B. et al. Remote sensing techniques to assess active fire characteristics and post-fire effects. International. Journal of Widland Fire, v.15, p.319-345, 2006.

MENDES-LOPES, J. M. C.; VENTURA, J. M. P.; AMARAL, J. M. P. Flame characteristics, temperature-time curves, and rate of spread in fire propagating in a bed of Pinus pinaster needles.

International Journal of Wildland Fire, v.12, n.1, p.67-84, 2003.

RIBEIRO, G. A. et al. Eficiência de um retardante de longa duração na redução da propagação do fogo. Revista Arvore, v.30, n.6, p.1025-1031, 2006

RIBEIRO, G. A.; SOARES, R. V. Estudo do comportamento da queima controlada em um povoamento de Eucalyptus viminalis. Revista Árvore, v.23, n.1, p.57-64, 1999.

ROTHERMEL, R. C. A matematical model for predicting fire spread in wildland fires. Ogden: USDA Forest Service, 1972. 41p. (Research Paper INT-115).

SANTONI, P. A. et al. Instrumentation of wildland fire: Characterization of a fire spreading through a Mediterranean shrub. Fire Safety Journal, v. 41, p 171-184, 2006.
SOARES, R. V.; BATISTA, A. C. Incêndios florestais - Controle, efeitos e uso do fogo. Curitiba: Independentes, 2007. 264p.

SOARES, R. V.; BATISTA, A. C.; NUNES, J. R. S. Incêndios florestais no Brasil o estado da arte. Curitiba: Independentes, 2009. 246p.

SOARES, R. V.; HAKKILA, P. Potencial energético dos resíduos de desbastes em plantações de Pinus taeda no estado do Paraná. Revista Florestal., v.17, n.1/2, p.73-94, 1987.

VEGA, J. A. et al. Predicting fire behaviour in Galicia (NW Spain) shrubland fuel complexes. In: VIEGAS, D. X., ed. INTERNATIONAL

CONFERENCE ON FOREST FIRE RESEARCH, 3., 1998, Luso. Proceedings... Coimbra: University of Coimbra, 1998. p.713-728.

VEGA, J. A. et al. Fire spread analysis of early summer field experiments in shrubland fuel types of Northwestern Iberia. In: VIEGAS, D. X., (Ed.) INTERNATIONAL CONFERENCE ON FOREST FIRE RESEARCH, 5., 2006, Figueira da Foz. Proceedings... Coimbra: University of Coimbra, 2006. p.S191.

VÈLEZ, R. La lucha contra los incendios forestales: Fundamentos y experiencias. Madrid: McGraw Hill, 2000. 750p. 
